\title{
Optimization of culture conditions and cultivation phase for the growth of Salvia viridis transformed roots and polyphenolic compound production
}

\author{
Izabela Grzegorczyk-Karolak ${ }^{1}$ D
}

Received: 2 April 2020 / Accepted: 1 July 2020 / Published online: 6 July 2020

(c) The Author(s) 2020

\begin{abstract}
Salvia viridis transformed root culture was studied using MS, WP or B5 media (full or half-strength macro- and microelements: $1 / 2 \mathrm{MS}, 1 / 2 \mathrm{WP}, 1 / 2 \mathrm{~B} 5)$ under varying light conditions to optimize growth and polyphenolic compound production. The highest levels of root growth ( $154.6 \mathrm{~g} / \mathrm{l}$ and $13.6 \mathrm{~g} / \mathrm{l}$, respectively, fresh and dry weight) were observed for hairy roots grown in WP medium in the dark. UPLC analysis found the polyphenolic acid content in plant material to range from 20.7 to $45.9 \mathrm{mg} / \mathrm{g}$ dry weight (DW) depending on the conditions. The highest metabolite content was observed in roots grown in B5 medium under photoperiods. A time-course study of the hairy roots cultured in WP medium in the dark examined the effect of culture period on biomass and polyphenolic compound yield every 5 days over a 60-day period. More than $1 \mathrm{~g}$ of dry weight per flask and $47.8 \mathrm{mg}$ of metabolite per g DW were produced after 30 days, at the beginning of the stationary phase. However, maximum production was only observed for four of the 10 detected compounds during this growth phase, between days 30 and 40; one of which was rosmarinic acid: the predominant compound in all extracts. Under optimal conditions, production was almost 10 times higher than that observed in the roots of field growing plants, which suggests that the selected optimized transformed root culture of $S$. viridis may be a good approach for valuable polyphenol production.
\end{abstract}

\section{Key Message}

The present study reports the influence of medium type, light condition and culture period on the accumulation of phenolic acids in hairy roots of $S$. viridis.

Keywords Growth curve $\cdot$ Medium optimization $\cdot$ Rosmarinic acid $\cdot$ Salvianolic acids $\cdot$ Salvia viridis

\section{Introduction}

Salvia viridis $\mathrm{L}$. has long been used as a herb in Turkey and Iran, especially against inflammatory and infectious diseases (Mosaddegh et al. 2012). Several classes of secondary metabolites have been isolated from the aboveground and underground parts of the plant, including polyphenolic acids, phenylethanoids, flavonoids (Rungsimakan and Rowan 2014; Grzegorczyk-Karolak and Kiss 2018), triterpenes (Ulubelen

Communicated by Ali R. Alan.

Izabela Grzegorczyk-Karolak

izabela.grzegorczyk@umed.lodz.pl

1 Department of Biology and Pharmaceutical Botany, Medical University of Lodz, Muszyńskiego 1, 90-151 Lodz, Poland et al. 1977), diterpenoids (Ulubelen et al. 2000) and essential oil (Kokkalou et al. 1982). The major component of $S$. viridis roots, rosmarinic acid (RA), is an ester of caffeic acid and 3,4-dihydroxyphenyllactic acid. Its biological activity has been extensively examined for many years, and many publications have confirmed its antioxidant (Furtado et al. 2010), antiviral (Abd-Elazem et al. 2002), antibacterial (Bais et al. 2002) and anti-inflammatory effects (Kuhlmann and Rohl 2006), as well as its antitumor potential (Anusuya and Manoharan 2011). Hamaguchi et al. (2009) found RA to play a protective role in the development of Alzheimer's disease by affecting various amyloid- $\beta$ aggregation pathways in vivo. Additionally, some of products used in the pharmaceutical, cosmetic, and food industries are based on RA (Psotova et al. 2006; Kim et al. 2010). 
Hairy root culture of $S$. viridis found to be a potentially rich source of valuable polyphenolic acids, such RA and salvianolic acids (Grzegorczyk-Karolak et al. 2018). In a selected clone (K3), RA level was found to be 8-fold higher than in the roots of field-grown plants. This culture was also characterized by high levels of salvianolic acids $\mathrm{E}$ and $\mathrm{F}$, which are found only in small quantities in the roots of field-grown $S$. viridis. Salvianolic acids are produced by some Salvia species such as S. miltiorrhiza (Ho and Hong 2011), and are well known for their effect on the alleviation of fibrosis and the treatment of cancer (Ma et al. 2019). In vivo and in vitro experiments have demonstrated that salvianolic acids promote the apoptosis of cancer cells and inhibit the epithelial-mesenchymal transition processes associated with cancer.

It is possible that highly-productive transformed root cultures of $S$. viridis may constitute an alternative approach to the commercial production of these important secondary metabolites. Such hairy root cultures, obtained by infection of plants with Agrobacterium rhizogenes strains grow quickly, are genetically stable and characterized by high production efficiency; they hence represent an attractive alternative to whole plant cultivation as sources of commercially-important secondary products. So far, transformed roots have been established from more than 100 medicinal plant species (Lan et al. 2015). Enhanced production of various phenolic acids, including rosmarinic acid, has been reported in hairy root cultures of Agastache rugosa (Lee et al. 2008), Ocimum basilicum (Srivastava et al. 2016), Salvia officinalis (Grzegorczyk et al. 2006), S. miltiorrhiza (Xing et al. 2018) or Coleus forskohlii (Li et al. 2005).

A previous study detailed the transformation procedure of $S$. viridis, and the selection of transformed clones according to their growth and bioactive metabolite production during 5-week culture in WP medium (GrzegorczykKarolak et al. 2018). However, although such production in transformed roots is determined mostly by genetic factors, it may also be influenced by the nutritional and environmental factors associated with the culture conditions. Salvia viridis shoot culture grown in the optimized medium was also found to proliferate more effectively and produce significantly increased levels of pharmacologically-active compounds (Grzegorczyk-Karolak et al. 2020). The aim of the present study is therefore to determine the optimal culture conditions for the most productive transformed root clone selected by Grzegorczyk-Karolak et al. (2018). It examines the effect of different media and light condition on hairy root growth and metabolite biosynthesis, and performs a detailed analysis of the biosynthesis of bioactive compounds every 5 days over the 60 -day culture period.

\section{Materials and methods}

\section{Plant material}

Hairy roots of $S$. viridis were obtained using A. rhizogenes strain A4 as described earlier (Grzegorczyk-Karolak et al. 2018), with clone $\mathrm{K} 3$, demonstrating the highest biomass and bioactive compound accumulation being selected for study (Grzegorczyk-Karolak et al. 2018). The roots were grown in $300 \mathrm{ml}$ Erlenmeyer flasks containing $80 \mathrm{ml}$ WP (Lloyd and McCown 1980) liquid medium on the rotary shaker at $70 \mathrm{rpm}$ at $24{ }^{\circ} \mathrm{C}$. Subcultures were carried out every 35 days.

\section{Basic medium selection}

To investigate the effects of liquid media on culture growth and metabolite biosynthesis, the hairy roots were transferred into flasks containing $80 \mathrm{ml}$ of liquid medium: MS (Murashige and Skoog 1962), WP or B5 (Gamborg et al. 1968 ) with full or half-strength macro- and microelements concentrations (1/2MS, 1/2WP, 1/2B5). The cultures were maintained on a rotary shaker at $70 \mathrm{rpm}$; they were kept either in the dark or under a photoperiod $(16 \mathrm{~h} \mathrm{light} / 8 \mathrm{~h}$ dark) with a light intensity of $40 \mu \mathrm{m} / \mathrm{m}^{2} \mathrm{~s}$. After 35 days, three flasks for each medium type and light condition were harvested to determine the accumulation of culture biomass and bioactive metabolites. Culture growth was determined for fresh (FW) and dry weight (DW), expressed as growth index (GI), as follows: $\mathrm{GI}=($ final biomass weight - initial biomass weight)/initial biomass weight. The experiments were repeated three times (passage 20-22).

\section{Growth and production studies}

To determine growth over time, hairy root culture $(0.57 \pm 0.023 \mathrm{~g} \mathrm{FW}$ and $0.047 \pm 0.002 \mathrm{~g} \mathrm{DW})$ was cultivated in $80 \mathrm{ml}$ of WP medium in $300 \mathrm{ml}$ flasks under dark conditions for 60 days. During this time, three flasks were harvested every 5 days and their FW, DW and polyphenolic compound content were evaluated. The experiments were repeated three times (passage 23-25).

\section{Extraction and UPLC determination of polyphenolic compound content}

Polyphenolic compounds were extracted and quantified according to Grzegorczyk-Karolak et al. (2018). Briefly, $100 \mathrm{mg}$ of lyophilized hairy roots was ground and ultrasonicated in methanol:water (8:2) solution for $15 \mathrm{~min}$ three times. Analysis was performed using an Agilent 
1290 Infinity UPLC system equipped with UV detector and an autosampler and Zorbax Eclipse Plus $\mathrm{C}_{18}$ column $(3 \times 100 \mathrm{~mm}, 1.8 \mu \mathrm{m}$; Agilent Technologies, USA). Ultrapure water containing $0.5 \%$ phosphoric acid (A) and acetonitrile containing $0.5 \%$ phosphoric acid (B) were used as chromatographic eluents. The gradient elution was programmed as follows: $0-22 \mathrm{~min} 6-26 \% \mathrm{~B} ; 22-27 \mathrm{~min} 26-90 \% \mathrm{~B}$; $27-27.5 \mathrm{~min} 90-95 \% \mathrm{~B}$ and $27.5-29 \mathrm{~min} 95 \% \mathrm{~B}$. The flow rate was $0.3 \mathrm{ml} / \mathrm{min}$, and the injection volume was $2 \mu \mathrm{l}$.

The compounds were identified on the basis of MS spectra, UV absorption spectra and retention time as described earlier (Grzegorczyk-Karolak et al. 2018). For quantitative analysis, standard calibration curves were constructed based on the peak area. Reference standards for rosmarinic acid, caffeic acid and salvianolic acid B were purchased from Sigma Aldrich, USA. The compounds with no available commercial standard were quantified as equivalents of a similar standard as described earlier (Grzegorczyk-Karolak et al. 2018). The results were expressed as $\mathrm{mg} / \mathrm{g}$ of DW. All analyses for all treatments were repeated three times.

\section{Statistical analysis}

The results are presented as mean values \pm standard error (SE). The significance of differences between treatments in the experiments was determined by analysis of variance (ANOVA), followed by the post hoc Tukey's test. A 5\% level of significance was assumed for all analyses. Statistical analysis was performed using Statistica 10.0 for Windows (StatSoft, Inc., Poland).

\section{Results and discussion}

\section{Basic medium selection}

Six different media (WP, B5, MS, 1/2WP, 1/2B5, 1/2MS) were tested under dark and photoperiod conditions to optimize the growth of $S$. viridis transformed root culture. The most productive clone identified by Grzegorczyk-Karolak et al. (2018), i.e. root clone K3, was used for the testing. The transformed nature of the culture was confirmed by PCR (polymerase chain reaction): amplification products of bacterial $r o l \mathrm{~B}$, rolC and $a u x 1$ genes were observed in the plant material (Grzegorczyk-Karolak et al. 2018).

The biomass and the morphology of transformed roots found to be significantly influenced by the type of medium and culture condition (Figs. 1,2). The highest growth indexes were achieved by transformed roots grown in the dark in WP medium: FW 26 and DW 28 (Fig. 3a, b). In this medium, mean hairy root biomass was $12.37 \pm 0.29 \mathrm{~g}$ FW and $1.09 \pm 0.038 \mathrm{~g}$ DW per flask after 5 weeks. Halving the macro and micro-element content in this medium $(1 / 2 \mathrm{WP})$ resulted in an approximate $40 \%$ drop in growth index. The roots grown in the WP and 1/2WP media, regardless of lighting conditions, were long and had numerous side branches (Fig. 2). Although the roots in the 1/2WP medium

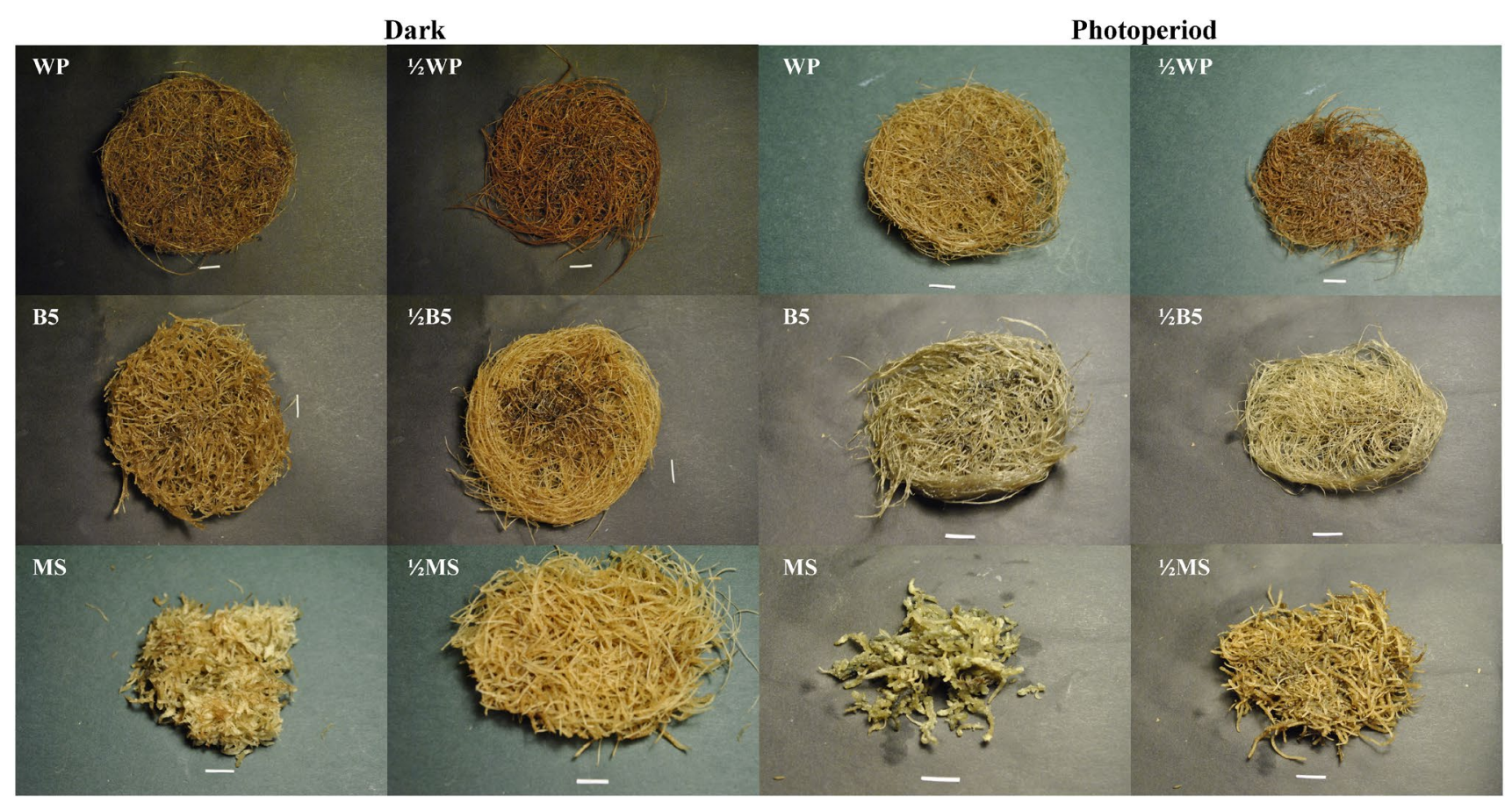

Fig. 1 Transformed roots of $S$. viridis K3 clone grown in WP, B5, MS, 1/2WP, 1/2B5 and 1/2MS media after 5 weeks (scale $1 \mathrm{~cm}$ ) 


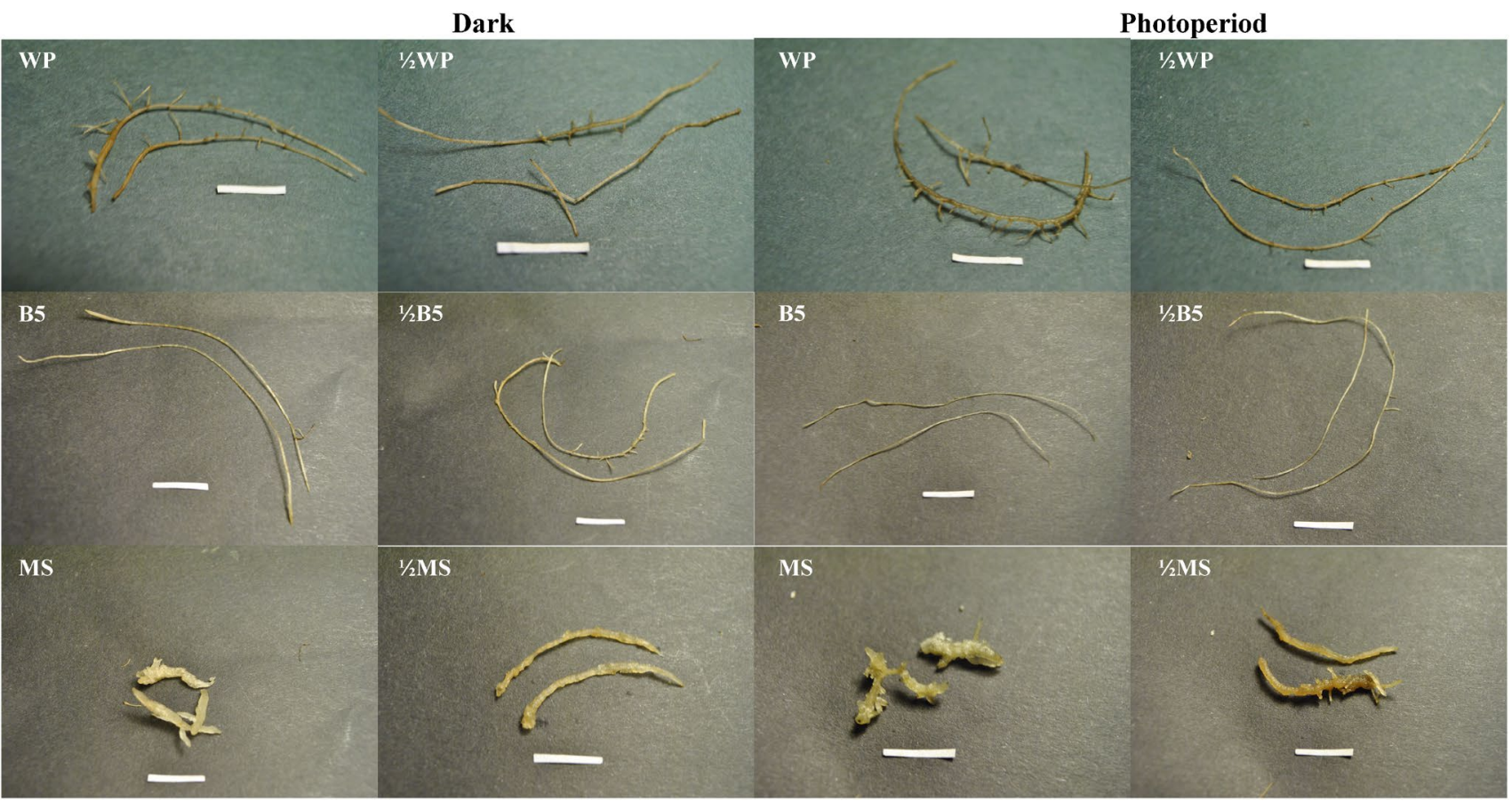

Fig. 2 Morphology of the individual transformed roots of $S$. viridis K3 clone in WP, B5, MS, 1/2WP, 1/2B5 and 1/2MS media after 5 weeks (scale $1 \mathrm{~cm}$ )

Fig. 3 Effect of medium composition on $S$. viridis culture fresh (a) and dry (b) weight expressed as growth index (GI). Hairy root were cultured under $16 \mathrm{~h}$ photoperiod or in dark for 5 weeks. The results are mean values \pm SE of nine replicates for each plant material. Different letters for the same parameter mean significant statistical differences between samples according to the one-way ANOVA, followed by the post hoc Tukey's test for multiple comparisons at $p<0.05$
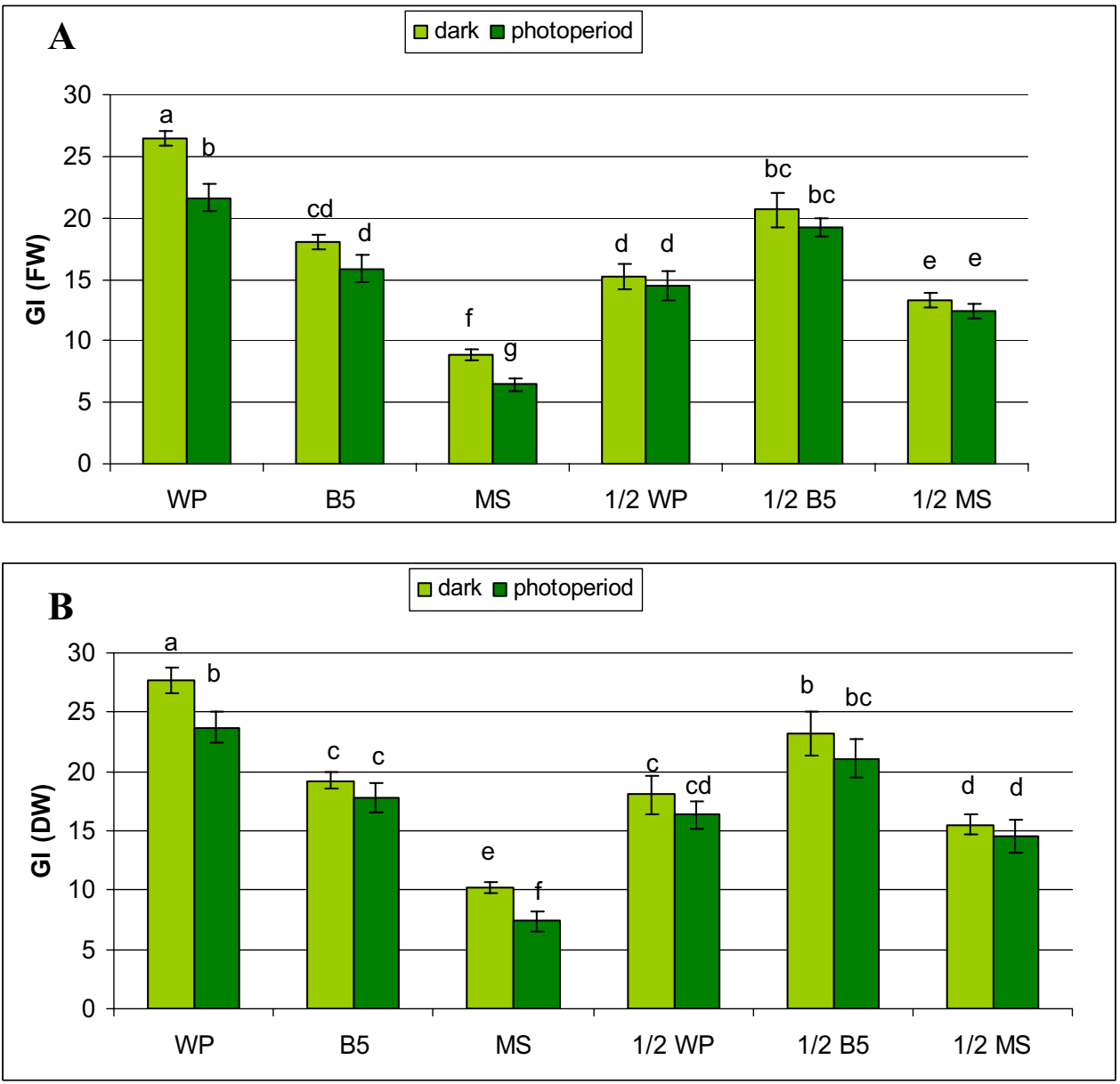
were slightly shorter than those in the WP medium, they branched more strongly, and their branches were longer. The roots grown in WP and 1/2WP medium were brown in color, although those grown in the light were slightly brighter (Fig. 1).

The hairy roots grown in the B5 and 1/2B5 media were brighter, yellowish, and those cultivated in the light were a cream color (Fig. 1). Both the B5 and 1/2B5-cultivated roots were long and relatively thin with poorly visible, but numerous and very short branches (Fig. 2). They demonstrated higher growth indexes than the roots grown in 1/2WP medium, but lower than those in optimal WP medium (Fig. 3a, b).

The least favorable medium for culture growth was found to be MS medium, with GI values of 9 for FW and 10 for DW. The roots grown in MS medium were light cream in color (Fig. 1), very short, and with short and thick branches (Fig. 2), they hence formed an unstable structure that easily disintegrated. They were particularly thick due to the formation of callus tissue and possibly by the high water content in root cells, as evidenced by the relatively low $\%$ dry weight of these roots compared to those grown in other media. A similar observation was noted for Hyoscyamus niger (Uchida et al. 1993), which was found to develop thick roots during cultivation in MS medium, but thin ones in WP and B5 medium. In the present study, the cultures grown in $1 / 2 \mathrm{MS}$ medium were thick in the middle part, but thinner and longer on the outside; therefore, they did not separate so easily and grew better than those in MS medium (Figs. 1,2).

Our results indicate that MS medium may not suitable for the growth of $S$. viridis hairy roots. It has the highest ionic strength of the tested media, because it is characterized by the highest content of macro- and micronutrients. Greater growth of $S$. viridis roots was observed for the cultures performed in 1/2MS medium, with reduced ionic strength than MS, and optimal growth was associated with WP medium, with the lowest macro- and microelement content among base media. Previous studies have also reported greater biomass accumulation of transformed Mirabilis himalaica roots at lower salt concentrations (Lan et al. 2015), and that WP also appeared to be the best choice of medium for the growth of transformed roots of H. niger (Uchida et al. 1993), Cephaelis ipecacuanha (Yoshimatsu et al. 2003) or Trigonella foenum-graecum (Merkli et al. 1997). In the latter case, the dry mass of roots was $606 \mathrm{mg} /$ flask (50 ml of medium) after 5 weeks of culture in WP medium; this value being 4 times higher than that observed in 1/2MS medium $(160 \mathrm{mg}$ /flask; $50 \mathrm{ml}$ of medium). However, in the present study, further reduction of macro- and micronutrient content, as observed in $1 / 2 \mathrm{WP}$, was no longer beneficial for $S$. viridis roots and resulted in a decrease in biomass accumulation (Fig. 3).

Nitrogen compounds are known to be important components of media, and ones that modify the culture response.
However, the consensus in the literature indicates that the ratio of different nitrogen sources has a greater impact on hairy root growth than total nitrogen concentration per se. The effect of this ratio on hairy root growth of Valeriana officinalis was evaluated by Parizo et al. (2015), who found the greatest biomass yield to be achieved by MS medium supplemented with a 20:20 ratio (mM) of $\mathrm{NH}_{4}{ }^{+}$to $\mathrm{NO}_{3}{ }^{-}$, followed by a 20:40 ratio. Meanwhile, Liu et al. (1997) report that the optimum $\mathrm{NH}_{4}{ }^{+}$to $\mathrm{NO}_{3}{ }^{-}$ratio for the growth of Artemisia annua hairy roots was 5:25. The hairy roots of Datura candida did not grown at all without $\mathrm{NH}_{4}{ }^{+}$. However, concentrations of ammonium ions higher than $10 \mathrm{mM}$ also inhibited culture growth (Nussbaumer et al. 1998); the optimal concentration of $\mathrm{NH}_{4}^{+}$for D. candida, was found to be $2 \mathrm{mM}$, present in $\mathrm{B} 5$ medium. Some researchers attribute the low culture growth response on MS medium to its high ammonium concentration (Danesh et al. 2006; WeremczukJeżyna et al. 2013) Oksman-Caldentey et al. (1994). showed that the ammonium is utilized before nitrate, leading to a decrease in the $\mathrm{pH}$ of the medium, which can inhibit culture growth. MS medium contains significantly larger amounts of ammonium nitrogen than WP. Changing the MS medium to $1 / 2 \mathrm{MS}$ and lowering the concentration of ammonium ions in the medium visibly improved the growth of $S$. viridis transformed roots.

Most studies indicate that micronutrient concentrations have little influence on culture growth. For example, increasing the amount of $\mathrm{Fe}^{2+}, \mathrm{Ca}^{2+}$ or $\mathrm{Zn}^{2+}$ had little effect on the growth of transformed roots of Hyosyamus albus (Christen et al. 1992). On the other hand, an increase in medium $\mathrm{Cu}^{2+}$ concentration stimulated the growth of the culture. Therefore, the fact that the concentration of $\mathrm{Cu}^{2+}$ in the WP medium was much higher than in the other media may have been an important factor for the growth of $S$. viridis hairy roots.

Whitney (1996) reported that $10 \mathrm{mg} / \mathrm{l}$ thiamine hydrochloride significantly stimulated the growth of hairy roots of red beet and tobacco; when the cultures grew slowly on MS medium without the vitamin. However, no data exists on the impact of $0.1 \mathrm{mg} / \mathrm{l}$ thiamine, the low concentrations present in standard MS medium, on culture growth, and the minimum amount needed to ensure the effective growth of transformed red beet and tobacco roots remains unknown. Moreover, while $10 \mathrm{mg} / \mathrm{l}$ of thiamin hydrochloride added to standard MS and 1/2MS media supported the growth of hairy roots of Picrorhiza kurroa (Verma et al. 2015), no change in the growth was observed when added to standard B5 medium. This confirms the fact that supplementation with vitamins beyond the optimal requirement level for any specific culture is ineffective. The content of thiamine in MS medium $(0.1 \mathrm{mg} / \mathrm{l})$ is far smaller than in B5 medium $(10 \mathrm{mg} / \mathrm{l})$ and WP medium $(1 \mathrm{mg} / \mathrm{l})$, which may explain the 
greater increases in biomass of hairy root $S$. viridis cultivated in the two latter media.

Hence, the small amount of ammonium nitrogen, and the high levels of thiamine and copper ions in medium could probably be the most significant factors supporting the growth of $S$. viridis hairy roots in WP medium.

Cultures grown in the light tended to display reduced biomass accumulation than those in the dark, although not all differences were statistically significant (Fig. 3). In addition, light grown cultures had a lighter color than those grown in the dark, but no significant differences in morphology were observed (Figs. 1, 2). Most root cultures described in previous studies, such as Levisticum officinale (Santos et al. 2005), S. officinalis (Grzegorczyk et al. 2006) or Dracocephalum moldavica (Weremczuk-Jeżyna et al. 2013), are usually performed in the dark; light does not tend to have a major impact on root growth, or could even inhibit it.

Variations in medium composition and the light environment also affected the polyphenolic compound content in the hairy root cultures, with the metabolite level ranging from 20.7 to $45.9 \mathrm{mg} / \mathrm{g}$ DW (Table 1 ). The greatest polyphenolic compound content was found in hairy roots grown in B5 medium under photoperiod conditions (Fig. 4). Rosmarinic acid predominated, constituting about $80 \%$ of the production of the 10 identified compounds. It was found in its greatest amounts, i.e. more than $35 \mathrm{mg} / \mathrm{g} \mathrm{DW}$, in B5 and WP media under photoperiod condition; this value was about 8 times higher than observed in the roots of soil-grown plants. Slightly lower levels were observed in the dark.

Table 1 Comparative productivity of total polyphenolic acids (TPA) in $S$. viridis hairy roots after 35 days in different liquid media in the dark and under photoperiod condition

\begin{tabular}{lll}
\hline Medium & Light condition & TPA content $(\mathrm{mg} / \mathrm{l}$ culture $)$ \\
\hline WP & Dark & $583.7 \pm 3.95 \mathrm{a}$ \\
& Photoperiod & $503.7 \pm 4.00 \mathrm{~b}$ \\
B5 & Dark & $421.0 \pm 5.60 \mathrm{~d}$ \\
& Photoperiod & $441.5 \pm 1.54 \mathrm{c}$ \\
MS & Dark & $197.6 \pm 2.61 \mathrm{~h}$ \\
& Photoperiod & $106.1 \pm 1.08 \mathrm{i}$ \\
1/2WP & Dark & $341.5 \pm 2.93 \mathrm{e}$ \\
& Photoperiod & $218.1 \pm 4.44 \mathrm{~g}$ \\
1/2B5 & Dark & $367.9 \pm 10.47 \mathrm{e}$ \\
& Photoperiod & $419.1 \pm 7.67 \mathrm{~d}$ \\
$1 / 2 \mathrm{MS}$ & Dark & $253.0 \pm 4.13 \mathrm{f}$ \\
& Photoperiod & $262.5 \pm 6.59 \mathrm{f}$ \\
\hline
\end{tabular}

The results are mean values \pm SE of nine replicates for each plant material. Different letters mean significant statistical differences between samples according to the one-way ANOVA, followed by the post hoc Tukey's test for multiple comparisons at $p<0.05$
Changes in medium composition and, to a lesser degree, lighting conditions significantly modified the metabolic pathways of the culture and its metabolite contents. WP medium in the dark, was optimal for the production of caffeic acid (0.5 mg/g DW) and salvianolic acid E $(2.6 \mathrm{mg} / \mathrm{g}$ DW); 1/2WP medium in the dark was optimal for salvianolic acid $\mathrm{J}(0.74 \mathrm{mg} / \mathrm{g} \mathrm{DW})$ and rosmarinic acid hexosides $(0.34$ and $0.57 \mathrm{mg} / \mathrm{g} \mathrm{DW}$, respectively); 1/2B5 medium under photoperiod was optimal for salvianolic acids $\mathrm{F}$ (1.9 and $4.1 \mathrm{mg} / \mathrm{g}$ DW, respectively); 1/2MS medium in the dark was optimal for methyl rosmarinate $(1.7 \mathrm{mg} / \mathrm{g}$ DW) (Fig. 4). Previous studies indicate that the optimal medium for growth may not to be the best for the production of individual bioactive compounds. For example, Lithospermum erythrorhizon transformed roots accumulated most biomass in B5 medium, but produced the most shikonin in MS medium (Hwang et al. 2002). T. foenum-graecum hairy roots grew most quickly in WP medium, but accumulated far greater diosgenin content in 1/2WP than in WP, B5 and 1/2B5 media, with no diosgenin accumulation observed in MS medium (Merkli et al. 1997).

As with the growth of cultures, the production of bioactive compounds could be affected by the nitrogen content in the medium. Peng et al. (2008) suggested that nitrogen sources act as signaling molecules, regulating the expression of a variety of genes. For example, it was reported that nitrogen depletion activated several PAL isozymes in plants, which strongly stimulated the biosynthesis of polyphenols, among others (Lillo et al. 2008). According Bryant et al. (1983), higher compound accumulation in some plant species can be explained by the carbon/nutrient balance hypothesis. Limited nitrogen availability can lead to a decrease in the biosynthesis of nitrogen-based secondary metabolites, but an increase in the accumulation of carbon-based secondary metabolites, such as polyphenols. Lowering nitrogen concentration in the MS medium from 60 to $40 \mathrm{mM}$ significantly enhanced polyphenol production in callus cultures of Abelmoschus esculentus (Irshad et al. 2018). B5 medium containing lower nitrogen concentration was found to be significantly more effective for the optimum level of iridioid glycoside production in hairy roots of $P$. kurroa than MS medium (Verma et al. 2015). After further reducing the concentration of macro- and micro elements (1/2B5 medium), this medium even more stimulated the biosynthesis of the bioactive metabolites. Oksman-Caldentey et al. (1994) also reported better growth and metabolite production when transformed Hyoscyamus muticus roots were cultivated in B5 medium than MS medium.

In addition, under limited nitrogen concentrations, Reay et al. (1998) observed chlorophyll and starch accumulation in the photosynthetic membranes, making them more sensitive to light, which may affect the production of certain groups of plant metabolites. Light is a factor 

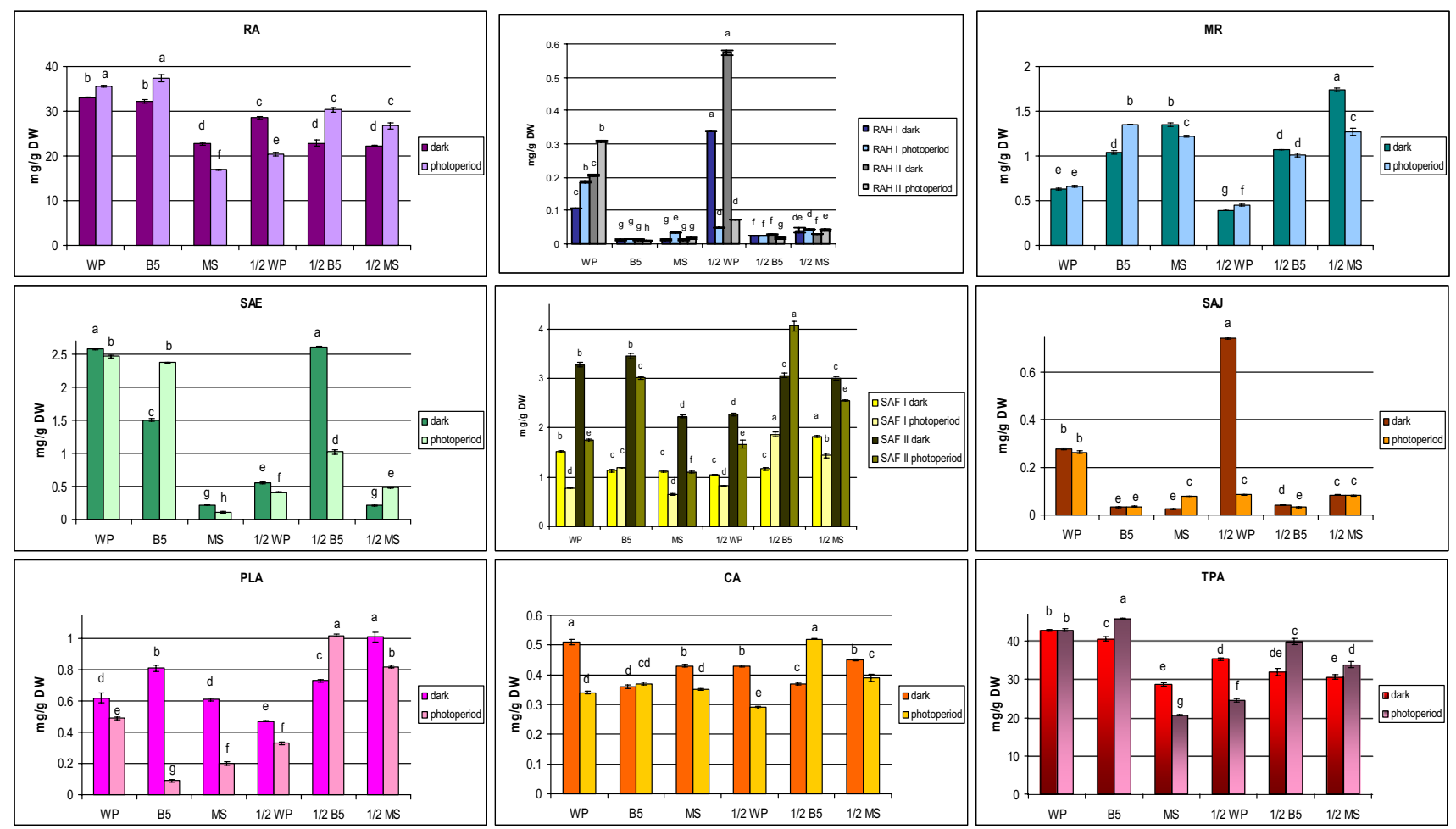

Fig. 4 Polyphenolic acid content in hairy roots of $S$. viridis cultured in different liquid media for 35 days. $R A$ rosmarinic acid, $R H I$ rosmarinic acid hexoside (I), $R H$ II rosmarinic acid hexoside (II), $M R$ methyl rosmarinate, $S A E$ salvianolic acid E, $S A F I$ salvianolic acid $\mathrm{F}$ (I), SAF II salvianolic acid F (II), SAJ salvianolic acid J, PLA prolithospermic acid, $C A$ caffeic acid, TPA total polyphenolic acids. The results are mean values \pm SE of nine replicates for each plant material. Different letters for the same metabolite mean significant statistical differences between samples according to the one-way ANOVA, followed by the post hoc Tukey's test for multiple comparisons at $p<0.05$

\section{Growth studies}

The transformed roots were cultured in WP medium over a 60-day period. Any increase in biomass and metabolite accumulation was recorded at 5-day intervals (Figs. 5, 6). The hairy roots began to grow on day 5 and reached the stationary phase on day 30 (Fig. 5). Maximum fresh and dry weights were obtained on day 35: $13.23 \pm 0.23 \mathrm{~g} \mathrm{FW/}$ flask and $1.05 \pm 0.018 \mathrm{~g}$ DW/flask. The values represent a 23 -fold increase of the inoculum biomass, i.e. $165.4 \mathrm{~g} \mathrm{FW}$ and $13.1 \mathrm{~g} \mathrm{DW}$ in 11 of medium over 35 days. To obtain an equal root mass from field grown plants, it is necessary to collect about 15 plants, as the dry weight of the roots of a single plant is only approximately $0.84 \mathrm{~g}$ after the growing season (about 4 months). The root system of this annual plant is poorly developed, consisting of only a small number of weakly-branched roots.

Initially after transfer into fresh medium, the production of most metabolites clearly dropped, usually by day 10 (Fig. 6). After this time, biosynthesis increased until a maximum was reached, usually in the stationary phase, suggesting that metabolite biosynthesis in the culture is related to its growth. The maximum total polyphenol content $(47.8 \mathrm{mg} / \mathrm{g}$ 


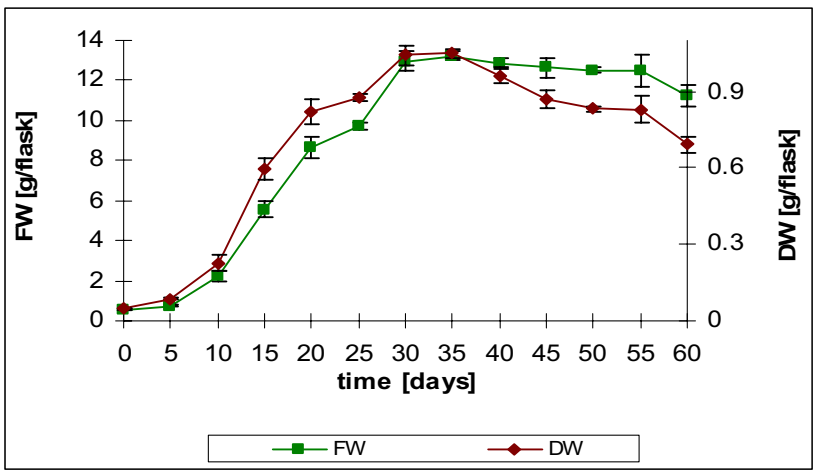

Fig. 5 Time course of biomass accumulation (fresh and dry weight) in $S$. viridis hairy roots cultured for 60 days in WP medium in dark. The results are means values $\pm \mathrm{SE}$ of nine replicates for each plant material

DW) was found in hairy roots of $S$. viridis on day 30 . However, only a few of the individual compounds, such as RA, its methyl ester, caffeic acid and prolithospermic acid reached their highest levels between 30 and 40 days, when the dry and fresh culture biomass was the highest (Fig. 6). It is often reported that metabolite biosynthesis appears to be synchronized with culture growth and maximum production occurs after reaching the stationary phase. This was the case for the transformed roots of Echinaceae purpurea, where the maximum production of all polyphenolic acids detected in the raw material (cichoric acid, caftaric acid, caffeic acid and chlorogenic acid) was achieved on the 40-45 day of culture, when the culture entered the stationary phase (Liu et al. 2006). A similar relationship has been also described for diosgenin synthesis in the roots of T. foenum-graecum (Merkli et al. 1997), emodin and physcion level in roots of Polygonum multiflorum (Thiruvengadam et al. 2014) or total isoflavonoid content in roots of Pueraria candollei (Danphitsanuparn et al. 2012). However, in the latter case, as for the transformed roots of $S$. viridis described here, the maximum production of some individual compounds falls outside the stationary phase.

The production of salvianolic acids such as SAF I, SAF II and SAE peaked before the stationary phase, i.e. between day 15 for SAE and day 25 for SAF I. Meanwhile SAJ was the only compound for which maximum biosynthesis was noted shortly after transfer of transformed roots into fresh medium, this being at the beginning of the growth cycle (day 5 of culture). In contrast, rosmarinic acid hexoside (I and II) levels were the highest after day 55: respectively, 0.188 and $0.303 \mathrm{mg} / \mathrm{g} \mathrm{DW}$; at this point,
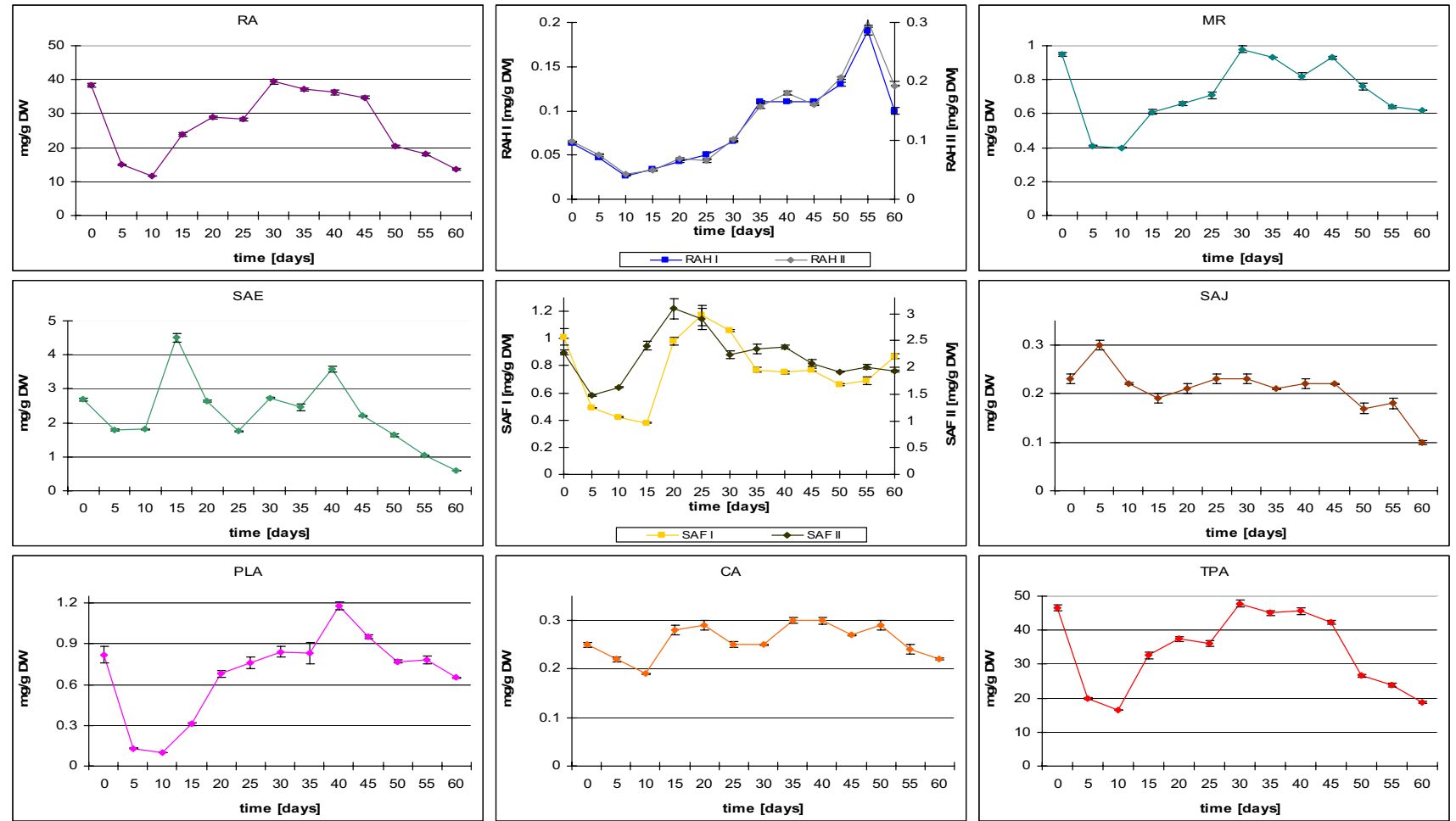

Fig. 6 Time-course study of polyphenolic acid production in S. viridis hairy roots cultured for 60 days in WP medium in dark. $R A$ rosmarinic acid, $R H I$ rosmarinic acid hexoside (I), $R H$ II rosmarinic acid hexoside (II), MR methyl rosmarinate, SAE salvianolic acid E,
SAF I salvianolic acid F (I), SAF II salvianolic acid F (II), SAJ salvianolic acid J, PLA prolithospermic acid, $C A$ caffeic acid, TPA total polyphenolic acids. The results are mean values \pm SE of nine replicates for each plant material 
the dry weight and levels of other compounds had already significantly decreased, and the total metabolite content was half that observed on day 30 (Fig. 6). This may indicate that the glycosylation of rosmarinic acid in the tissues of the plant occurs only at a later time, after culture growth had ended, and this may be associated with the accumulation of large amounts of RA. This would be consistent with the previous observations that these derivatives were not found, or were observed only in trace amounts, in the roots of plants grown in the field conditions showing a quite different growth cycle, where the level of RA was 8-fold lower (Grzegorczyk-Karolak et al. 2018).

Only slight fluctuations in caffeic acid (CA) were observed across the whole culture period, with levels ranging from 0.19 to $0.3 \mathrm{mg} / \mathrm{g}$ DW. This relatively constant level may suggest that the emerging CA is commonly used as a substrate in the synthesis of more complex polyphenolic compounds. Similar levels of CA were found in the roots of intact plants.

Regarding the changes in biomass observed during the growth period, the productivity of the culture was found to be $623.6 \mathrm{mg} / \mathrm{l}$ after 30 days of cultivation. After this time, productivity decreased, due to both a decrease in metabolite biosynthesis and a gradual loss of culture biomass. It would be necessary to collect almost 150 plants to get a comparable amount of polyphenolic acid from roots of field-grown plants, and greater time would be needed to get this amount, i.e. about 4 months.

Our findings demonstrate that $S$. viridis hairy root culture, transformed with $A$. rhizogenes, is a valuable system for studying the biosynthesis of polyphenolic acids in the plant. The choice of basal medium had a significant influences on biomass and bioactive metabolite accumulation in the transformed roots. The greatest total phenolic acid content $(47.8 \mathrm{mg} / \mathrm{g} \mathrm{DW})$ was obtained by culture in WP medium for 30 days; this level is almost 10 times higher than detected in the roots of 4-month-old non-transformed plants grown in the field. The results of these studies indicate that an optimized culture of $S$. viridis hairy roots can be a promising basis for attempts to increase the scale of their cultivation, thus providing an efficient source of bioactive metabolites.

Acknowledgements This study was supported by Medical University of Lodz, a Grant No. 503/3-012-01/503-31-001-19-00. Special thanks to Dr. Ł Kuźma (Medical University of Lodz) for technical support in UPLC analysis.

Author contributions IG-K designed and performed the experiments, analyzed the data, wrote the paper and is responsible for final content of the manuscript.

Open Access This article is licensed under a Creative Commons Attribution 4.0 International License, which permits use, sharing, adaptation, distribution and reproduction in any medium or format, as long as you give appropriate credit to the original author(s) and the source, provide a link to the Creative Commons licence, and indicate if changes were made. The images or other third party material in this article are included in the article's Creative Commons licence, unless indicated otherwise in a credit line to the material. If material is not included in the article's Creative Commons licence and your intended use is not permitted by statutory regulation or exceeds the permitted use, you will need to obtain permission directly from the copyright holder. To view a copy of this licence, visit http://creativecommons.org/licenses/by/4.0/.

\section{References}

Abd-Elazem IS, Chen HS, Bates RB, Huang RC (2002) Isolation of two highly potent and non-toxic inhibitors of human immunodeficiency virus type 1 (HIV-1) integrase from Salvia miltiorrhiza. Antivir Res 55:91-106. https://doi.org/10.1016/s0166 -3542(02)00011-6

Anusuya C, Manoharan S (2011) Antitumor initiating potential of rosmarinic acid in 7,12-dimethylbenz(a)anthracene-induced hamster buccal pouch carcinogenesis. J Environ Pathol Toxicol Oncol 30:199-211. https://doi.org/10.1615/jenvironpatholtoxico loncol.v30.i3.30

Bais HP, Walker TS, Schweizer HP, Vivanco JM (2002) Root specific elicitation and antimicrobial activity of rosmarinic acid in hairy root cultures of Ocimum basilicum. Plant Physiol Biochem 40:983-995. https://doi.org/10.1016/S0981-9428(02)01460-2

Bryant JP, Chapin FS III, Klein DR (1983) Carbon/nutrient balance of boreal plants in relation to vertebrate herbivory. Oikos 40(3):357-368. https://www.jstor.org/stable/3544308

Christen P, Aoki T, Shimomura K (1992) Characteristics of growth and tropane alkaloid production in Hyoscyamus albus hairy roots transformed with Agrobacterium rhizogenes A4. Plant Cell Rep 11(12):597-600. https://doi.org/10.1007/BF00236380

Danesh YR, Galtapeh EM, Alizadeh A (2006) Study on the growth patterns of transformed carrot hairy roots in on optimized system. J Agric Technol 2:89-97

Danphitsanuparn P, Boonsnongcheep P, Boriboonkaset T, Chintapakorn Y, Prathanturarug S (2012) Effects of Agrobacterium rhizogenes strains and other parameters on production of isoflavonoids in hairy roots of Pueraria candollei Grah. ex Benth. Var. candollei. Plant Cell Tissue Organ Cult 111(3):315-322. https://doi.org/10.1007/s11240-012-0196-8

Furtado RA, de Araújo FR, Resende FA, Cunha WR, Tavares DC (2010) Protective effect of rosmarinic acid on V79 cells evaluated by the micronucleus and comet assays. J Appl Toxicol 30:254-259. https://doi.org/10.1002/jat.1491

Galbiati M, Chiusi A, Peterlongo P, Gavazzi G, Mancinelli A (1994) Photoinduction of anthocyanin in maize; a genetic approach. Maydica 39:89-95

Gamborg OL, Miller RA, Ojina K (1968) Nutrient requirement of suspension cultures of soybean root cell. Exp Cell Rep 1968:151-158. https://doi.org/10.1016/0014-4827(68)90403-5

Grzegorczyk-Karolak I, Kiss AK (2018) Determination of the phenolic profile and antioxidant properties of Salvia viridis L. shoots: a comparison of aqueous and hydroethanolic extracts. Molecules 23:1468. https://doi.org/10.3390/molecules23061468

Grzegorczyk-Karolak I, Królicka A, Wysokińska H (2006) Establishment of Salvia officinalis L. hairy root cultures for the production of rosmarinic acid. Z Nat 61c:351-356. https://doi. org/10.1515/znc-2006-5-609

Grzegorczyk-Karolak I, Kuźma Ł, Skała E, Kiss AK (2018) Hairy root cultures of Salvia viridis L. for production of polyphenolic compounds. Ind Crops Prod 117:235-244. https://doi. org/10.1016/j.indcrop.2018.03.014 
Grzegorczyk-Karolak I, Hnatuszko-Konka K, Zarzycka M, Kuźma $Ł$ (2020) The stimulatory effect of purine-type cytokinins on proliferation and polyphenolic compound accumulation in shoot culture of Salvia viridis. Biomolecules 10:178. https:// doi.org/10.3390/biom 10020178

Hamaguchi T, Ono K, Murase A, Yamada M (2009) Phenolic compounds prevent Alzheimer's pathology through different effects on the amyloid-beta aggregation pathway. Am J Pathol 175:2557-2565. https://doi.org/10.2353/ajpath.2009.090417

Ho JH, Hong CY (2011) Salvianolic acids: small compounds with multiple mechanisms for cardiovascular protection. J Biomed Sci 18:30. https://doi.org/10.1186/1423-0127-18-30

Hwang OJ, Kim YJ, Sung NS, Ahn JC, Kim SE, Hwang B (2002) Optimization of major culture elements on growth and shikonin production in the Lithospermum erythrorhizon hairy root culture. Korean J Med Crop Sci 10:243-248

Irshad M, Debnath B, Mitra S, Arafat Y, Li M, Sun Y, Qiu D (2018) Accumulation of anthocyanin in callus cultures of red-pod okra [Abelmoschus esculentus (L.) Hongjiao] in response to light and nitrogen levels. Plant Cell Tissue Organ Cult 134(1):29-39. https://doi.org/10.1007/s11240-018-1397-6

Kim HJ, Kim TH, Kang KC, Pyo HB, Jeong HH (2010) Microencapsulation of rosmarinic acid using polycaprolactone and various surfactants. Int J Cosmet Sci 32:185-191. https://doi.org/10.11 11/j.1468-2494.2010.00526.x

Kokkalou E, Koedam A, Phokas G (1982) Composition of the essential oil of Salvia horminum L. (Labiatae). Pharm Acta Helv 57:317-320

Kuhlmann A, Rohl C (2006) Phenolic antioxidant compounds produced by in vitro cultures of rosemary Rosmarinus officinalis and their anti-inflammatory effect on lipopolysaccharide activated microglia. Pharm Biol 44:401-410. https://doi. org/10.1080/13880200600794063

Lan X, Quan H, Xia X, Yin W (2015) Establishment of hairy root cultures and analysis of rotenoid in Tibetan medicinal plant 'Mirabilis himalaica'. Plant Omics 8:335

Lee S, Xu H, Kim Y, Park S (2008) Rosmarinic acid production in hairy root cultures of Agastache rugosa Kuntze. World J Microbiol Biotechnol 24:969-972. https://doi.org/10.1007/ s11274-007-9560-y

Li W, Koike K, Asada Y, Yoshikawa T, Nikaido T (2005) Rosmarinic acid production by Coleus forskohlii hairy root cultures. Plant Cell Tissue Organ Cult 80:151-155. https://doi.org/10.1007/ s11240-004-9541-x

Lillo C, Lea US, Ruoff P (2008) Nutrient depletion as a key factor for manipulating gene expression and product formation in different branches of the flavonoid pathway. Plant Cell Environ 31:587-601. https://doi.org/10.1111/j.1365-3040.2007.01748.x

Liu CZ, Wang YC, Ouyang F, Ye HC, Li GF (1997) Production of artemisinin by hairy root cultures of Artemisia аппиа L. Biotechnol Lett 19(9):927-929. https://doi.org/10.1023/A:10183 62309677

Liu CZ, Abbasi BH, Gao M, Murch SJ, Saxena PK (2006) Caffeic acid derivatives production by hairy root cultures of Echinacea purpurea. J Agric Food Chem 54(22):8456-8460. https://doi. org/10.1021/jf061940r

Lloyd GM, McCown BH (1980) Commercially-feasible micropropagation mountain laurel Kalmia latifolia by use of shoot-tip culture. Comb Proc Int Plant Propagator Soc 30:421-427

Ma L, Tang L, Yi Q (2019) Salvianolic acids: potential source of natural drugs for the treatment of fibrosis disease and cancer. Front Pharmacol 10:97. https://doi.org/10.3389/fphar.2019.00097

Merkli A, Christen P, Kapetanidis I (1997) Production of diosgenin by hairy root of Trigonella foenum-graecum L. Plant Cell Rep 16:632-636. https://doi.org/10.1007/BF01275505
Mosaddegh M, Naghibi F, Moazzeni H, Pirani A, Esmaeili S (2012) Ethnobotanical survey of herbal remedies traditionally used in Kohghiluyeh va Boyer Ahmad Province of Iran. J Ethnopharmacol 141:80-95. https://doi.org/10.1016/j.jep.2012.02.004

Murashige T, Skoog F (1962) A revised medium for rapid growth and bioassays with tobacco tissue cultures. Physiol Plant 15:473-497. https://doi.org/10.1111/j.1399-3054.1962.tb08052.x

Nussbaumer P, Kapétanidis I, Christen P (1998) Hairy roots of Datura candida $\times D$. aurea: effect of culture medium composition on growth and alkaloid biosynthesis. Plant Cell Rep 17(5):405-409. https://doi.org/10.1007/s002990050415

Oksman-Caldentey KM, Sevón N, Vanhala L, Hiltunen R (1994) Effect of nitrogen and sucrose on the primary and secondary metabolism of transformed root cultures of Hyoscyamus muticus. Plant Cell Tissue Org Cult 38(2-3):263-272. https://doi.org/10.1007/ BF00033886

Parizi AP, Farsi M, Nematzadeh GA, Mirshamsi A (2015) Impact of different culture media on hairy roots growth of Valeriana officinalis L. Acta Agric Slov 103(2):299-305. https://doi.org/10.14720 /aas.2014.103.2.14

Peng M, Hudson D, Schofield A, Tsao R, Yang R, Gu H, Bi Y-M, Rothstein SJ (2008) Adaptation of Arabidopsis to nitrogen limitation involves induction of anthocyanin synthesis which is controlled by the NLA gene. J Exp Bot 59:2933-2944. https://doi.org/10.1093/ jxb/ern 148

Psotova J, Svobodova A, Kolarova H, Walterova D (2006) Photoprotective properties of Prunella vulgaris and rosmarinic acid on human keratinocytes. J Photochem Photobiol B 84:167-174. https://doi. org/10.1016/j.jphotobiol.2006.02.012

Reay PF, Fletcher RH, Thomas V (1998) Chlorophylls, carotenoids and anthocyanin concentrations in the skin of 'Gala' apples during maturation and the influence of foliar applications of nitrogen and magnesium. J Sci Food Agric 76:63-71. https://doi.org/10.1002/ (SICI) 1097-0010(199801)76:1<63::AID-JSFA908>3.0.CO;2-K

Rungsimakan S, Rowan MG (2014) Terpenoids, flavonoids and caffeic acid derivatives from Salvia viridis L. cvar. Blue Jeans. Phytochemistry 108:177-188. https://doi.org/10.1016/j.phyto chem.2014.08.029

Santos PAG, Figueiredo AC, Oliveira MM, Barroso JB, Pedro LG, Deans SG, Scheffer JJC (2005) Growth and essential oil composition of hairy roots of Levisticum officinale W. D. J. Kooch (lavage). Plant Sci 168:1089-1096. https://doi.org/10.1016/j.plant sci.2004.12.009

Srivastava S, Conlan XA, Adholeya A, Cahill DM (2016) Elite hairy roots of Ocimum basilicum as a new source of rosmarinic acid and antioxidants. Plant Cell Tissue Organ Cult 126(1):19-32. https:// doi.org/10.1007/s11240-016-0973-x

Thiruvengadam M, Praveen N, Kim EH, Kim SH, Chung IM (2014) Production of anthraquinones, phenolic compounds and biological activities from hairy root cultures of Polygonum multiflorum Thunb. Protoplasma 251:555-566. https://doi.org/10.1007/s0070 9-013-0554-3

Uchida K, Kuroyanagi M, Ueno A (1993) Tropane alkaloid production in hairy roots of Hyoscyamus niger transformed with Agrobacterium rhizogenes. Plant Tissue Cult Lett 10:223-228. https://doi. org/10.5511/plantbiotechnology 1984.10.223

Ulubelen A, Brieskorn CH, Oezdemir N (1977) Triterpenoids of Salvia horminum, constitution of a new diol. Phytochemistry 16:790 791. https://doi.org/10.1016/S0031-9422(00)89266-3

Ulubelen A, Öksüz S, Kolak U, Bozok-Johansson C, Çelik C, Voelter W (2000) Antibacterial diterpenes from the roots of Salvia viridis. Planta Med 66:458-462. https://doi.org/10.1055/s-2000-8596

Verma PC, Singh H, Negi AS, Saxena G, Rahman LU, Banerjee S (2015) Yield enhancement strategies for the production of picroliv from hairy root culture of Picrorhiza kurroa Royle ex Benth. 
Plant Signal Behav 10(5):1023976. https://doi.org/10.1080/15592 324.2015.1023976

Weremczuk-Jeżyna I, Grzegorczyk-Karolak I, Frydrych B, Królicka A, Wysokińska H (2013) Hairy roots of Dracocephalum moldavica: rosmarinic acid content and antioxidant. Acta Physiol Plant 35:2095-2103. https://doi.org/10.1007/s11738-013-1244-7

Whitney PJ (1996) Hormone independent root organ cultures of rye (Secale cereale). Plant Cell Tissue Organ Cult 46(2):109-115. https://doi.org/10.1007/BF00034843

Xing B, Yang D, Liu L, Han R, Sun Y, Liang Z (2018) Phenolic acid production is more effectively enhanced than tanshinone production by methyl jasmonate in Salvia miltiorrhiza hairy roots. Plant
Cell Tissue Organ Cult 134(1):119-129. https://doi.org/10.1007/ s11240-018-1405-x

Yoshimatsu K, Shimomura K, Yamazaki M, Kiucki F (2003) Transformation of Ipecac (Cephaelis ipecacuanha) with Agrobacterium rhizogenes. Planta Med 69:1018-1023. https://doi. org/10.1055/s-2003-45149

Publisher's Note Springer Nature remains neutral with regard to jurisdictional claims in published maps and institutional affiliations. 